\title{
Use of Raman Spectroscopy in the Investigation of Debundling of Single Walled Carbon Nanotubes
}

\author{
Elizabeth Gregan \\ Technological University Dublin, Elizabeth.Gregan@tudublin.ie \\ Sinead Keogh \\ Technological University Dublin \\ Theresa Hedderman \\ Technological University Dublin, Theresa.Hedderman@tudublin.ie
}

See next page for additional authors

Follow this and additional works at: https://arrow.tudublin.ie/radcon

Part of the Physics Commons

\section{Recommended Citation}

Gregan, E., Keogh, S.M. \& Hedderman, T.G. (2005). Use of Raman spectroscopy in the investigation of debundling of single walled carbon nanotubes. Proceedings of Optolreland, 2005. SPIE, vol. 5826, pg.56. doi:10.1117/12.605062

This Conference Paper is brought to you for free and open access by the Radiation and Environmental Science Centre at ARROW@TU Dublin. It has been accepted for inclusion in Conference papers by an authorized administrator of ARROW@TU Dublin. For more information, please contact arrow.admin@tudublin.ie, aisling.coyne@tudublin.ie, gerard.connolly@tudublin.ie. 
Authors

Elizabeth Gregan, Sinead Keogh, Theresa Hedderman, Gordon Chambers, and Hugh J. Byrne

This conference paper is available at ARROW@TU Dublin: https://arrow.tudublin.ie/radcon/5 


\title{
Use of Raman Spectroscopy in the investigation of debundling of single walled carbon nanotubes
}

\author{
E. Gregan ${ }^{2 *}$, S.M. Keogh ${ }^{1,2}$, T.G. Hedderman², G. Chambers ${ }^{2}$, H.J. Byrne ${ }^{1}$, \\ FOCAS Institute ${ }^{1}$, School of Physics ${ }^{2}$, Dublin Institute of Technology, Kevin Street, Dublin 8, \\ Ireland.
}

\begin{abstract}
Samples of raw nanotubes are compared to those deposited from solutions to examine separation of nanotube bundles. Single wall nanotubes bundles produced by the arc-discharge and HiPco methods were solubilised in toluene, DMF and 1,2 dichloroethane. Resonant Raman spectroscopy was used to determine if debundling of the tubes sample occurred. The results showed some degree of debundling, best for the 1,2 dichloroethane solvent, which also shows long term solubility.
\end{abstract}

Keywords: Carbon Nanotubes, Raman, HiPco, Arc-discharge, Debundling, Solubility

\section{INTRODUCTION}

Carbon nanotubes, long, thin cylinders of carbon, were discovered in 1991 by S. Iijima ${ }^{[1]}$. These are large macromolecules that are unique for their size, shape, and remarkable physical properties. These intriguing structures have sparked much excitement in the recent years and a large amount of research has been dedicated to their understanding.

Carbon nanotubes are graphene sheets of $\mathrm{sp}^{2}$-bonded carbon arranged in a cylindrical formation. Single Walled Nanotubes are classified into two primary groups chiral and achiral. A nanotube is achiral if the mirror image of the nanotube has an identical structure to the original one. There are two types of achiral nanotubes; armchair and zigzag. These are formed by bisecting a $\mathrm{C}_{60}$ molecule at the equator and joining the two resulting hemispheres with a cylindrical tube one monolayer thick and with the same diameter as the $C_{60}$. If the $C_{60}$ molecule is bisected normal to a five-fold axis, the armchair nanotube is formed. If the $\mathrm{C}_{60}$ molecule is bisected normal to a threefold axis, the zigzag nanotube is formed. A chiral nanotube mirror image cannot be superimposed onto the original tube.

Different types of nanotubes can be described by the chiral vector $(n, m)$, where $n$ and $m$ are integers of the vector equation. The chiral vector is defined by ${ }^{[2]}$

$$
\mathbf{C}_{\mathrm{h}}=\mathrm{n} \mathbf{a}_{1}+\mathrm{ma} \mathbf{a}_{2}
$$

An ensemble of possible chiral vectors can be specified by above equation in terms of pairs of integers $(n, m)$ and this ensemble is shown in figure 1.

The values of $\mathrm{n}$ and $\mathrm{m}$ determine the chirality, or "twist" of the nanotube. The chirality in turn affects the conductance of the nanotube, its density, its lattice structure, and other properties. For all metallic nanotubes, independent of their diameter and chirality, it follows that the density of states per unit length, $N\left(E_{f}\right)$ along the nanotube axis is a constant given by ${ }^{[2]}$

$$
N\left(E_{f}\right)=\frac{8}{\sqrt{3 \pi a|t|}}
$$

where $\mathbf{a}$ is the lattice constant and $|\mathbf{t}|$ is the nearest neighbour carbon to carbon bond tight binding overlap energy. The equation shows the electronic $1 D$ density of states per unit cell of a $2 D$ metallic and semi-conducting graphene sheet. 
The density of states for the nanotubes at the Fermi energy, $\mathbf{E}_{\mathrm{f}}$ located at $\mathbf{E}_{\mathrm{f}}=0$, is zero for semi-conducting nanotubes, and is non-zero for metallic tubes. The energy gap $\mathbf{E}_{\mathrm{g}}$ for semi-conducting nanotubes depends on the reciprocal of the nanotube diameter $\mathbf{d}_{\mathrm{t}}$, which is independent of the chirality angle, and is given by ${ }^{[3]}$

$$
E_{g}=\frac{|t| a C-C}{d_{t}} .
$$

$\mathrm{C}-\mathrm{C}=\mathrm{a} / \sqrt{3}$ is the nearest neighbour $\mathrm{C}-\mathrm{C}$ distance on the graphene sheet. Experimental results have shown that one third of all nanotubes are conducting and the remaining two thirds are semi-conducting. At the Fermi energy (the highest occupied energy level), the density of states is finite for a metallic tube (though very small), and zero for semiconducting tubes as mentioned before. As the energy is increased, sharp peaks in the density of states, called Van Hove singularities, appear at specified energy levels, see Fig.2.

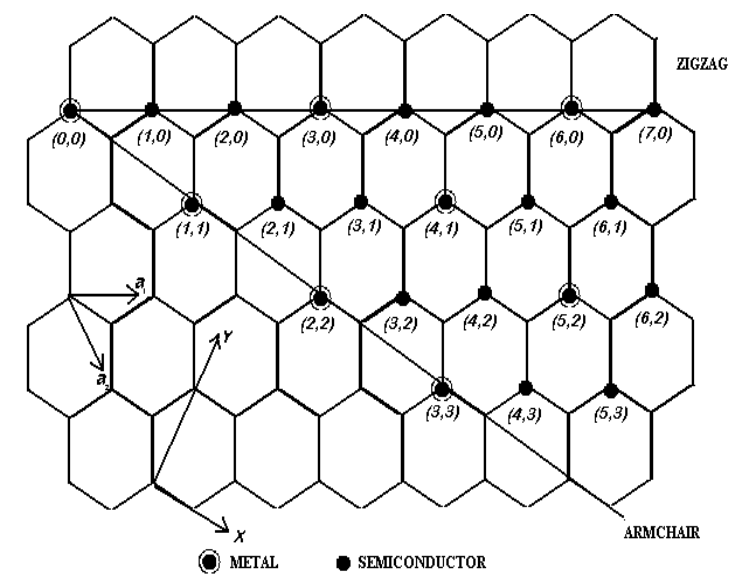

Fig.1 Graphene layer with atoms labelled using $(n, m)$ notation. Unit vectors of the 2D lattice
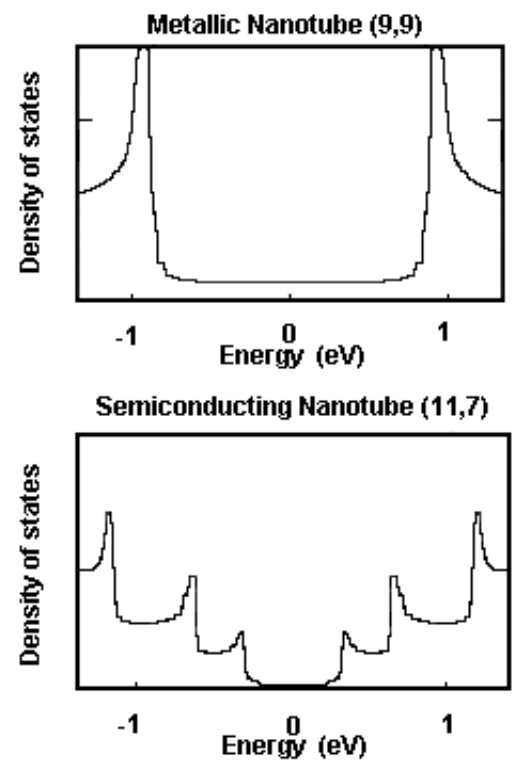
A SWNT is considered metallic if the value $\mathrm{n}-\mathrm{m}$ is divisible by three. Otherwise, the nanotube is semiconducting. Consequently, when tubes are formed with random values of $n$ and $m$, we would expect that two-thirds of nanotubes would be semi-conducting, while the other third would be metallic, which happens to be the case.

Each pair of integers $(n, m)$ defines a different way of rolling the graphene sheet to form a carbon nanotube. Given the chiral vector $(n, m)$, the diameter of a carbon nanotube can be determined using the relationship

$$
\mathrm{d}_{\mathrm{t}}=\sqrt{3} a_{c-c}\left(\mathrm{n}^{2}+\mathrm{m}^{2}+\mathrm{nm}\right)^{1 / 2} / \pi
$$

where $a_{c-c}$ is the nearest-neighbour C-C distance (bond length) ${ }^{[2]}$

Currently, the physical properties of SWNT are still being explored and disputed. What makes it so difficult is that nanotubes have a very broad range of electronic, thermal and structural properties that change depending on the different kinds of nanotube (defined by its diameter, length and chirality or twist), and this broad range potentially exists in any one sample. Nanotubes vary in size, depending on how they are produced (see later) and they aren't always perfectly cylindrical. The larger nanotubes, such as a $(20,20)$ tube, tend to bend under their own weight. The average bond length of 1.42 A was measured by Spires and Brown in 1996 and later confirmed by Wildöer in $1998^{[3]}$. The C-C tight bonding overlap energy is in the order of $2.5 \mathrm{eV}$. Wildöer estimated it to be between $2.6 \mathrm{eV}-2.8 \mathrm{eV}$ while at the same time, Odom $^{[4]}$ estimated it to be $2.45 \mathrm{eV}$.

The first Fig.2: Density of States diagram, showing Van Hove singularities for a metallic and semi-conducting tube

nanotubes were discovered while studying the material deposited on the cathode during the arc-evaporation synthesis of fullerenes ${ }^{[1]}$. It was found that the central core of the cathodic deposit contained a variety of closed graphitic structures including nanoparticles and nanotubes, of a type, which had never previously been observed. A short time later ${ }^{[5]}$, it was shown that nanotubes could be produced in bulk quantities by varying the arc-discharge evaporation conditions. This paved the way to an explosion of research into the physical and chemical properties of carbon nanotubes in laboratories all over the world. A major event in the development of carbon nanotubes was the synthesis in 1993 of single-walled nanotubes ${ }^{[6]}$. The standard arc-discharge method produces only multiwalled tubes. It was found that addition of metals such as cobalt to the graphite electrodes resulted in an extremely fine tube with a single-wall. An alternative method of preparing single-walled nanotubes was described in $1995^{[7]}$. Like the original method of preparing $\mathrm{C}_{60}$, this involved the laser-vaporisation of graphite, and resulted in a high yield of single-walled tubes with unusually uniform diameters. These highly uniform tubes had a greater tendency to form aligned bundles than those prepared using arc-evaporation. Initial analysis of the tubes produced by this method showed that the samples contained a very high proportion of nanotubes with a specific armchair structure however subsequent work showed that the samples were less homogeneous than originally thought. The HiPco process, high pressure CO disproportionation, has been shown to produce nanotubes of high purity, with current purity of $90 \%$ atomic percent SWNT ${ }^{[8,9,10]}$. The yield and diameter size can be varied by controlling the process parameters.

Another problem arises from the fact that nanotubes aggregate in bundles as a result of substantial van der Waals attractions between tubes. It has been observed that increased purity in a sample is associated with increased bundle size ${ }^{[11]}$. It is thought that there are fewer small particles present to interfere with the van der Waals attraction between neighbouring tubes. In 1996, Thess ${ }^{[12]}$ measured the properties of "ropes"of carbon nanotubes. On a macroscopic scale the bundles mat together. It was found that the individual SWNTs packed into a close-packed triangular lattice with a lattice constant of about $17 \AA$. In addition it was concluded that the density, lattice parameter, and interlayer spacing of the ropes was dependent on the chirality of the tubes in the mat. $(10,10)$ Armchair tubes had a lattice parameter of 16.78 $\AA$ and had a density of $1.33 \mathrm{~g} / \mathrm{cm}^{3}$. Zigzag tubes of the chirality $(17,0)$ had a lattice parameter of $16.52 \AA$ and a density of $1.34 \mathrm{~g} / \mathrm{cm}^{3}$. Mats made of $(12,6)$ chiral SWNTs had a lattice parameter of $16.52 \AA$ and a density of $1.40 \mathrm{~g} / \mathrm{cm}^{3}$. The space between the tubes and therefore the binding energy of the bundles is dependent on chirality and the diameter of the tubes in the bundles. Armchair tubes had a spacing of $3.38 \AA$, zigzag tubes had a spacing of $3.41 \AA$, and (2n, n) chiral tubes had an interlayer spacing value of $3.39 \AA$.

In this study comparison is made of the ability of different solvents to break up bundles of tubes. Two different samples of tubes were used, one produced by the arc-discharge method and one produced by the HiPco process. The diameter 
distribution of these sample have been previously determined to be 1.2 to $1.4 \mathrm{~nm}$ for arc-discharge sample and 0.7 to 1.3 $\mathrm{nm}$ for the HiPco sample ${ }^{[13][14]}$. Due to the higher purity it is expected that the HiPco samples will have a bigger bundle size than the arc-discharge tubes. In addition due to the smaller mean tube diameter the binding energy within the HiPco bundles will be stronger. The average bundle size for HiPco tubes has been found to $15-30 \mathrm{~nm}^{[15]}$.

Raman spectroscopy has been a very useful tool in the study of carbon nanotubes for some time ${ }^{[2]}$. The Raman spectrum of a single walled tube as seen in figure 3 shows three main regions. The $\mathrm{G}$-line which originates from the $\mathrm{C}=\mathrm{C}$ stretching mode in graphene, shows peaks around $1580 \mathrm{~cm}^{-1}$. This peak is doubly split into higher and lower sides of the $1580 \mathrm{~cm}^{-1}$ line, $\mathrm{G}^{+}$and $\mathrm{G}^{-}$, due to the curvature of the graphene sheet. The $\mathrm{G}^{+}$arises from the atomic displacements along the tube axis, and $\mathrm{G}^{-}$, for modes with atomic displacement along the circumferential direction.

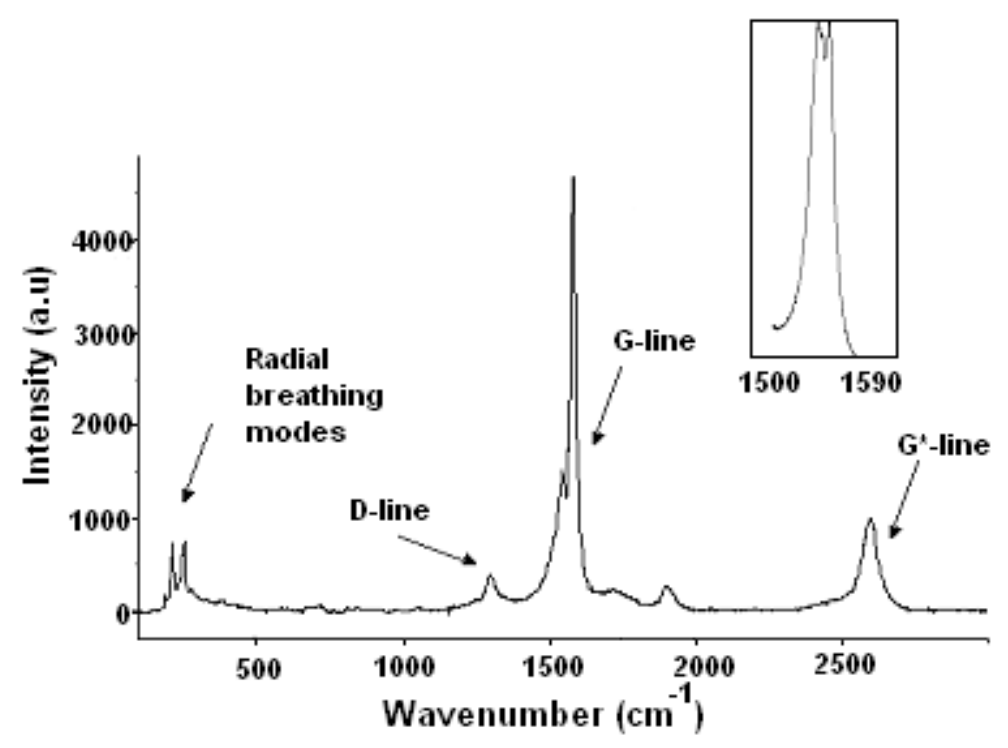

Fig. 3: Stokes Raman spectrum of semiconducting nanotube taken at $633 \mathrm{~nm}$ with inset of metallic nanotube G-line shape

G-Line

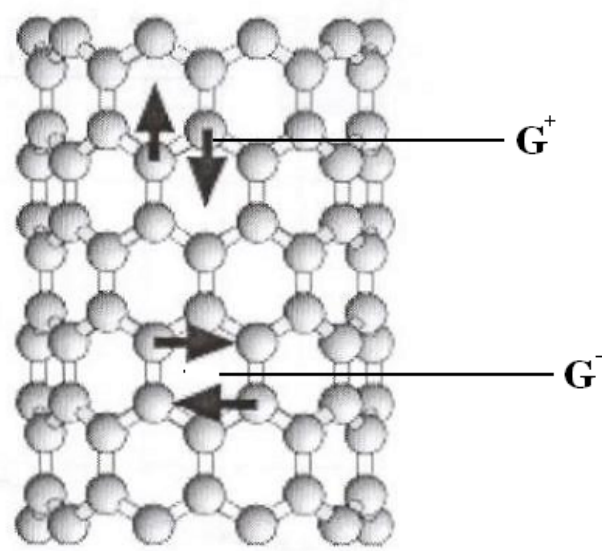

RBM

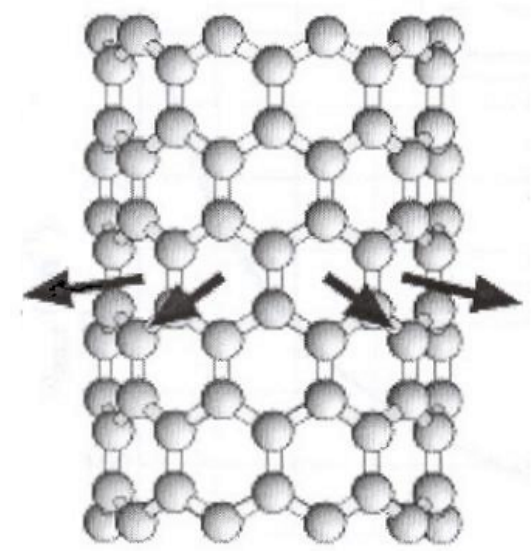

Fig. 4: Schematic picture showing the atomic vibrations for (a) G-line and (b) RBM modes 
The lineshape that results is indicative of the electronic nature of the tube. The semi-conducting tube produces a G-line which is Lorentzian in shape ${ }^{[16][17]}$. In metallic tubes the $\omega_{G}^{-}$feature is broadened. This broadening is related to the presence of free electrons in nanotubes with metallic character. The metallic nanotube shows a Breit-Wigner-Fano line shape $^{[18,19]}$. Examination of the G-line features for isolated tubes shows that the higher component $\omega_{G}^{+}$is essentially independent of tube diameter. However the lower component $\omega_{G}^{-}$is dependant on tube diameter and decreases in frequency in accordance with

$$
\omega_{G}^{-}=\omega_{G}^{+}-\frac{C}{d_{t}^{2}}
$$

where $d_{t}$ is the tube diameter, and $C=47.7 \mathrm{~cm}^{-1} \mathrm{~nm}^{2}$ for semiconducting tubes and $\mathrm{C}=79.5 \mathrm{~cm}^{-1} \mathrm{~nm}^{2}$ for metallic tubes. ${ }^{\text {[21] }}$. Th G-line for bundles also shows the two features whose spacing depends on the mean diameter of the nanotube bundle ${ }^{[.22]}$.

The linewidths $\Gamma_{\mathrm{G}^{+}}$for the G peaks from isolated SWNTs are usually around 5-15 $\mathrm{cm}^{-1}$, and the same range of linewidths for $\Gamma_{\mathrm{G}}$ are found for semiconducting isolated SWNTs ${ }^{[23]}$. For semiconducting SWNTs in bundles, the linewidths are related to the intertube interactions and to the diameter distribution. Therefore the broadening principally occurs for $\Gamma_{\mathrm{G}}$. For metallic SWNTs, the broadening is minor for $\Gamma_{\mathrm{G}^{+}}$, while for $\Gamma_{\mathrm{G}-}$ a significant broadening occurs, and it is found that the linewidth for the BWF line is strongly dependent on tube diameter. For isolated tubes with $\mathrm{d}_{\mathrm{t}}>2 \mathrm{~nm}, \Gamma_{\mathrm{G}}$ is similar to semiconducting SWNTs, and the line mostly looks like a normal Lorentzian, reflecting the small magnitude of the BWF effect. The BWF effect increases as the tube diameter decreases, causing the $\Gamma_{\mathrm{G}}$ feature to become more asymmetric and broad. Values of $\Gamma_{\mathrm{G}-}>70 \mathrm{~cm}^{-1}$ have been observed for isolated metallic SWNTs ${ }^{\text {[24] }}$.

The intensity of the D-line was thought until recently to be a measure of the amount of impurity in the sample. However more recently it is thought to be a measure of the level of defects along the tube itself. There is also some indication that the D-line resonance is effected by the electronic nature of the tubes ${ }^{[25]}$. It has also been shown that the D-line varies in both value and amplitude with laser excitation frequency ${ }^{[14]}$. The D-line and its second harmonic $\mathrm{G}^{*}$-line are highly dispersive and observed in isolated tubes and bundles. However in bundles superimposed on the linear dependence of $\omega_{\mathrm{D}}$ and $\omega_{\mathrm{G}^{*}}$ on laser energy is an oscillatory feature due to resonance of the laser energy with specific van Hove singularities ${ }^{[24]}$. The D-line intensity is large compared to that of the G-line when the sample has a large number of defects while the $\mathrm{G}^{*}$-line intensities always shows a large intensity comparable to the G-line without defects. Therefore the ratio of G/D line and $G^{*} / D$ line intensities provides a good index for showing the presence of defects decreasing with increasing order and purity ${ }^{[25]}$. The width of the D-line is smaller for semiconducting tubes than for metallic tubes.

In the lower frequency region the spectrum is dominated by the in-phase mode known as the radial breathing mode (RBM), see figure 3. The frequency of the RBM $\left(\omega_{\mathrm{RBM}}\right)$ is inversely proportional to the nanotube diameter $\left(d_{t}\right)$. For bundles

$$
\omega_{R B M}=\frac{A}{d_{t}}+B
$$

the spectral shift B being an empirically determined environmental damping factor. For isolated tubes

$$
\omega_{R B M}^{\prime}=\frac{A^{\prime}}{d_{t}}
$$

The spectral shape and position of the Raman band associated with the RBM of the nanotubes present in a sample provide an estimate of the diameter distribution in the SWNT sample ${ }^{[26]}$. It should be noted that tubes which are most apparent in a spectrum are those which are resonantly enhanced at the wavelength employed. This is because of the one 
dimensional density of states bands which are sharp and narrow. Therefore within a sample only some tubes are resonant at a given wavelength and in order to characterise fully a given sample, it is necessary to use a range of excitation frequencies. Most recently Wang et al have developed a method for assigning resonant scattering peaks in the Raman spectrum $^{[27]}$. From the radial breathing modes, data of diameters, RBM frequencies and electronic density of states have been used to graphically assign these peaks.

Lattice dynamical predictions indicate that isolated tubes should have lower $\omega_{\mathrm{RBM}}$ values than those in bundles.. However it has been observed that the frequencies actually upshift for single tubes in solution due to a decreased energy spacing of the Van Hove singularities in isolated tubes over the spacings in bundles thereby allowing the same laser excitation to excite different diameter tubes in the two samples ${ }^{[28]}$. The net effect of debundling is an apparent up-shift in the Raman active RBM's ${ }^{[29]}$.

\section{EXPERIMENTAL}

Raman measurements were taken using an Instruments S.A. Labram 1B. The Labram system is a confocal Raman imaging microscope system. Both a Helium-Neon laser $(632.8 \mathrm{~nm})$ and an external Argon ion 514.5nm laser were used as sources. Both lasers are polarised, enabling measurement of depolarisation ratios and studies of orientation in materials. The light is imaged to a diffraction limited spot (typically $1 \mu \mathrm{m}$ ) via the objective of an Olympus BX40 microscope. The scattered light is collected by the objective in a confocal geometry, and is dispersed onto an air cooled CCD array by one of two interchangeable gratings, 1800 lines $/ \mathrm{mm}$ or 600 lines $/ \mathrm{nm}$, allowing a range of $150 \mathrm{~cm}^{-1}$ to $4000 \mathrm{~cm}^{-1}$ to be covered in a single image, or with greater resolution in a combination of images. With the former, a spectral resolution of $1 \mathrm{~cm}^{-1}$ per pixel is achievable. The confocal, microscopic system allows measurement of powdered samples with no further sample preparation. Spectral X-Y mapping may be performed with a precision of $0.1 \mu \mathrm{m}$.

Solutions of $0.001 \mathrm{mg} / 30 \mathrm{mls}$ tubes to solvent ratio were made using arc-discharge and HiPco tubes. This is the concentration range where nanotubes in polymer composite solutions are reported to be debundled ${ }^{[30]}$. Three different solvents, toluene, DMF and 1,2 dichloroethane(DCE) were used. The solutions were sonicated using a sonic tip for 30 seconds, allowed to settle and decanted. Samples were then made by drop casting the solutions on glass slides. Raman spectra were the taken of these samples as well as spectra of the corresponding untreated raw tubes. The spectra were compared for changes.

\section{RESULTS AND DISCUSSION}

The first observation was the ease with which the tubes dissolved in the 1,2 dichlorethane. Even without sonication both the arc-discharge and HiPco tubes visibly when to solution. It should also be noted that several months later the tubes were still in solution in the DCE whereas they had long since fallen out of solution in the toluene and the DMF.

Spectra were obtained for a given laser line from many different points across the raw samples to see if the resulting spectra varied. Most variation in the resulting spectra was observed in the RBM regions. From figure 5 below it can be seen that although the relative amplitudes of the peaks varies in the spectra the same tubes are resonant at all times.

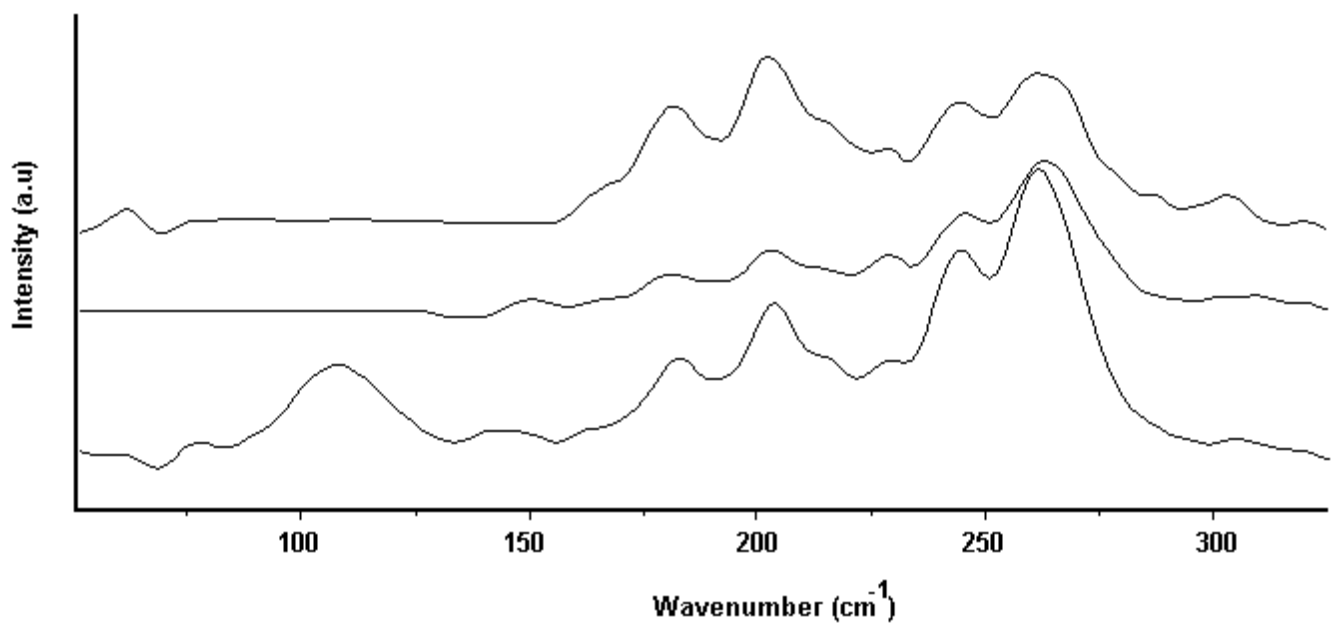

Fig 5: Stokes spectra of the RBM region for a number of spots across a HiPco sample at 514nm 
The spectra for the solvent treated samples were examined. Some parameters were measured from spectra and tabulated in table 1 and table 2.

\begin{tabular}{|l|c|c|c|c|}
\hline Intensity & Raw Tube & Toluene & DMF & DCE \\
\hline $\begin{array}{l}\text { G-line Solvent Sample/ Raw } \\
\text { Sample }\end{array}$ & - & 3.85 & 4.2 & 15 \\
\hline G-line/D-line ratio & 11.75 & 10.38 & 9.99 & 15.47 \\
\hline $\mathrm{G}^{*}$-line/D-line ratio & 2.76 & 2.8 & 1.77 & 2.21 \\
\hline$\Gamma_{\mathrm{G}}\left(\mathrm{cm}^{-1}\right)$ & 36 & 35 & 38 & 36 \\
\hline
\end{tabular}

Table 1: From Stokes spectra of HiPco samples at 633nm

\begin{tabular}{|l|c|c|c|c|}
\hline Intensity & Raw Tube & Toluene & DMF & DCE \\
\hline $\begin{array}{l}\text { G-line Solvent Sample/ Raw } \\
\text { Sample }\end{array}$ & - & 1.1 & 3.0 & 9.9 \\
\hline G-line/D-line ratio & 1.93 & 16.4 & 3.17 & 12.74 \\
\hline $\mathrm{G}^{*}$-line/D-line ratio & 0.66 & 9.10 & 0.89 & 4.5 \\
\hline$\Gamma_{\mathrm{G}}\left(\mathrm{cm}^{-1}\right)$ & 30 & 46 & 46 & 49 \\
\hline
\end{tabular}

Table 2: From Stokes spectra for arc-discharge samples at 633nm

It can be seen that for all solvents there was an increase in the intensity of the spectra for both types of tubes as evidenced in the G-line ratios above. This pattern was repeated for the $514 \mathrm{~nm}$ spectra. The increase was biggest for the DCE samples and smallest for toluene. This most probably results from a dispersion of the tubes into a less compact form. This can be simply a density effect or it could be a due to reduced damping as a result of debundling.

The G/D and G*/D ratios, which are a measure of the level of order/impurity in the samples show varying results. Worth noting are the large increases in the values for the arc-discharge toluene samples. This can be explained by the effect of the toluene on dissolving tubes allowing the other impurities to precipitate resulting in a reduction in the intensity of the D-line. No such increase is seen for the HiPco tubes, consistent with their inherent purity. Notably when the spectra for both the toluene samples are examined more closely apart from changes in intensity values these spectra show little deviation from the spectra of the raw tubes, (see figure 6), implying little debundling. So it can be concluded that in toluene, bundles are solubilised, precipitating impurities, but no real debundling occurs.

The spectra for arc-discharge and HiPco tubes in DCE at $633 \mathrm{~nm}$ are shown in figure7 and figure 8 below. In both cases there is evidence of an upshift in the spectra of the treated samples indicating some level of debundling. In the case of the HiPco tubes this is about 3-4 $\mathrm{cm}^{-1}$. The profile of the RBM region in the case of the arc-discharge tubes has altered. The peaks are more distinguished and spread out indicating that the tubes are less bound together allowing greater freedom of vibration resulting in more resolution of the resonant tubes. The profile of the G-line in the arc-discharge samples has also changed. In the raw tubes it shows a mixed character of semiconducting and metallic tubes. However in the treated samples this has become predominantly metallic. This would point towards the DCE having preferentially dissolving the metallic tubes rather than the semiconducting tubes.

In the case of the HiPco tubes, previous studies have shown little evidence of metallic tube resonances at either $633 \mathrm{~nm}$ or $514 \mathrm{~nm}$. There is thus little change in the G-line profile in those processed with DCE (figure 8). The RBM region 
similarly shows little difference to that of the raw sample. In the sample primarily consisting of semiconducting tubes, while the shift in the G-line may indicate some degree of debundling, there are few differences between the raw sample and the processed sample. The HiPco samples do however remain in solution over prolonged periods of time and thus it must be concluded that DCE dissolved the HiPco bundles. It is to be expected that due to their higher purity and smaller diameter size the binding within the HiPco bundles will be stronger than the binding within arc-discharge bundles. It therefore follows that it will be easier to break up the arc-discharge bundles than the HiPco as evidenced in the spectra.

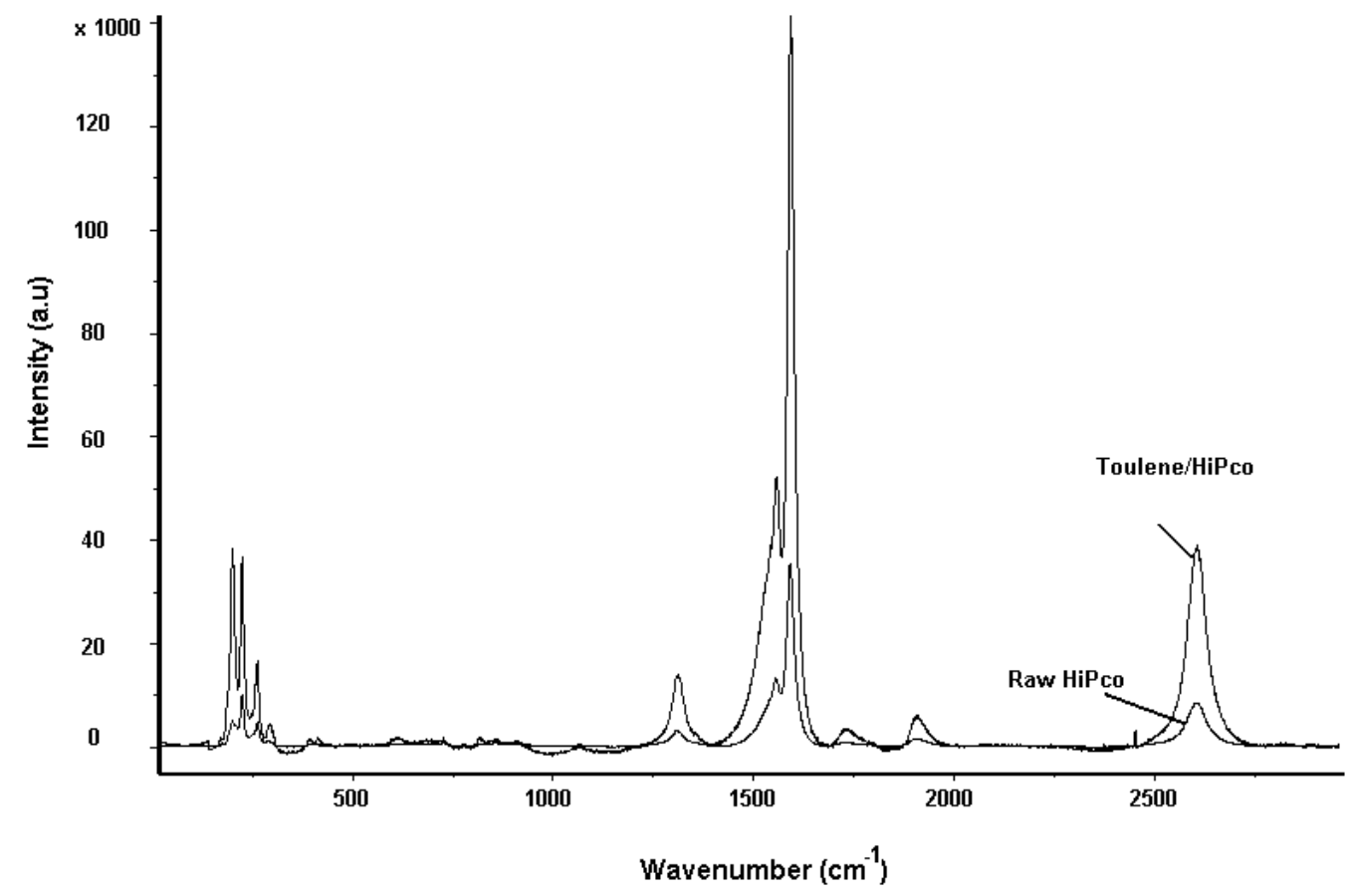

Fig. 6: Stokes Raman spectra HiPco and Toluene/ HiPco samples at 633nm

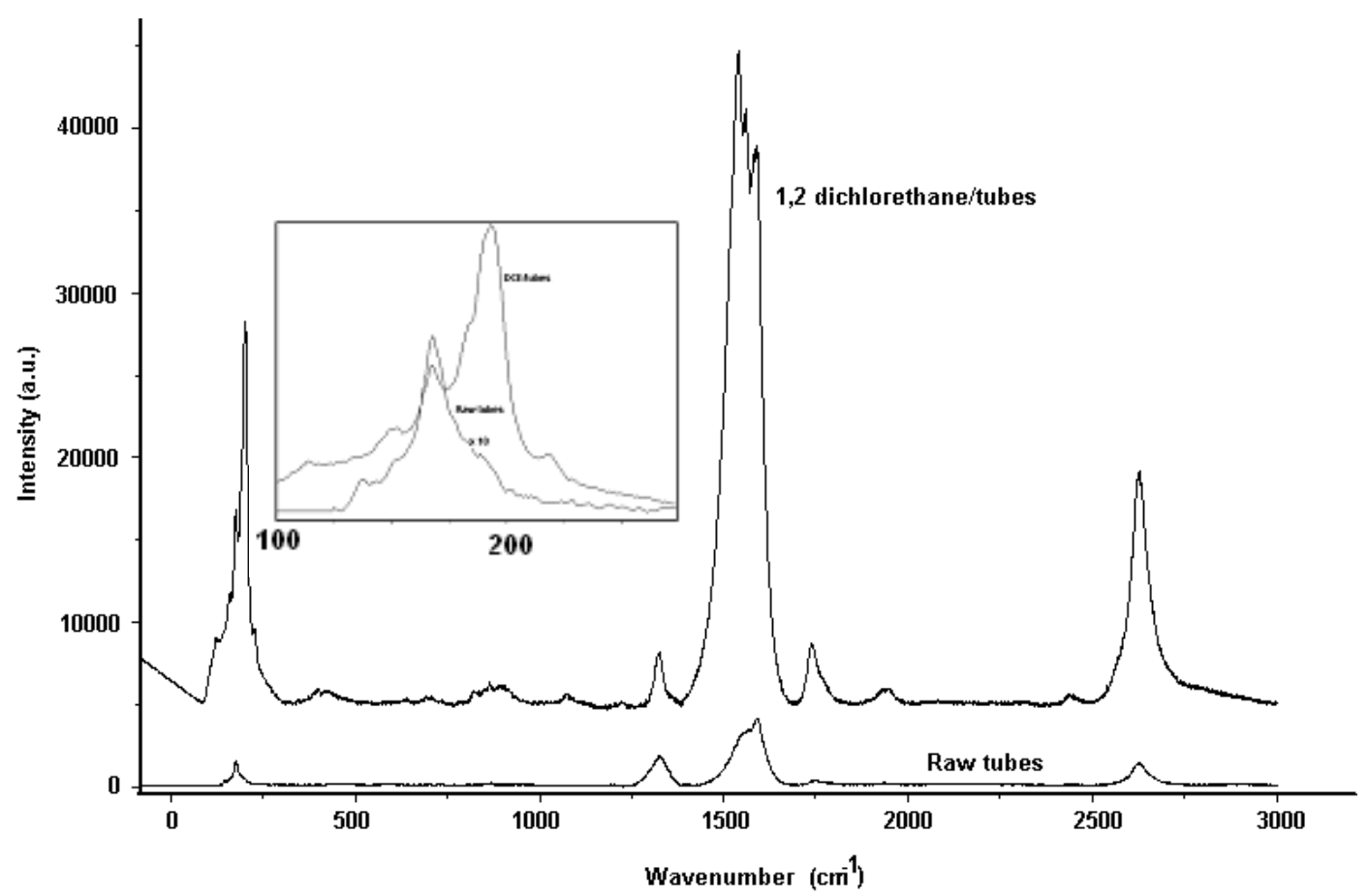

Fig. 7: Stokes Raman spectra for arc-discharge and DCE / arc-discharge samples at 633nm with inset of expanded RBM region 


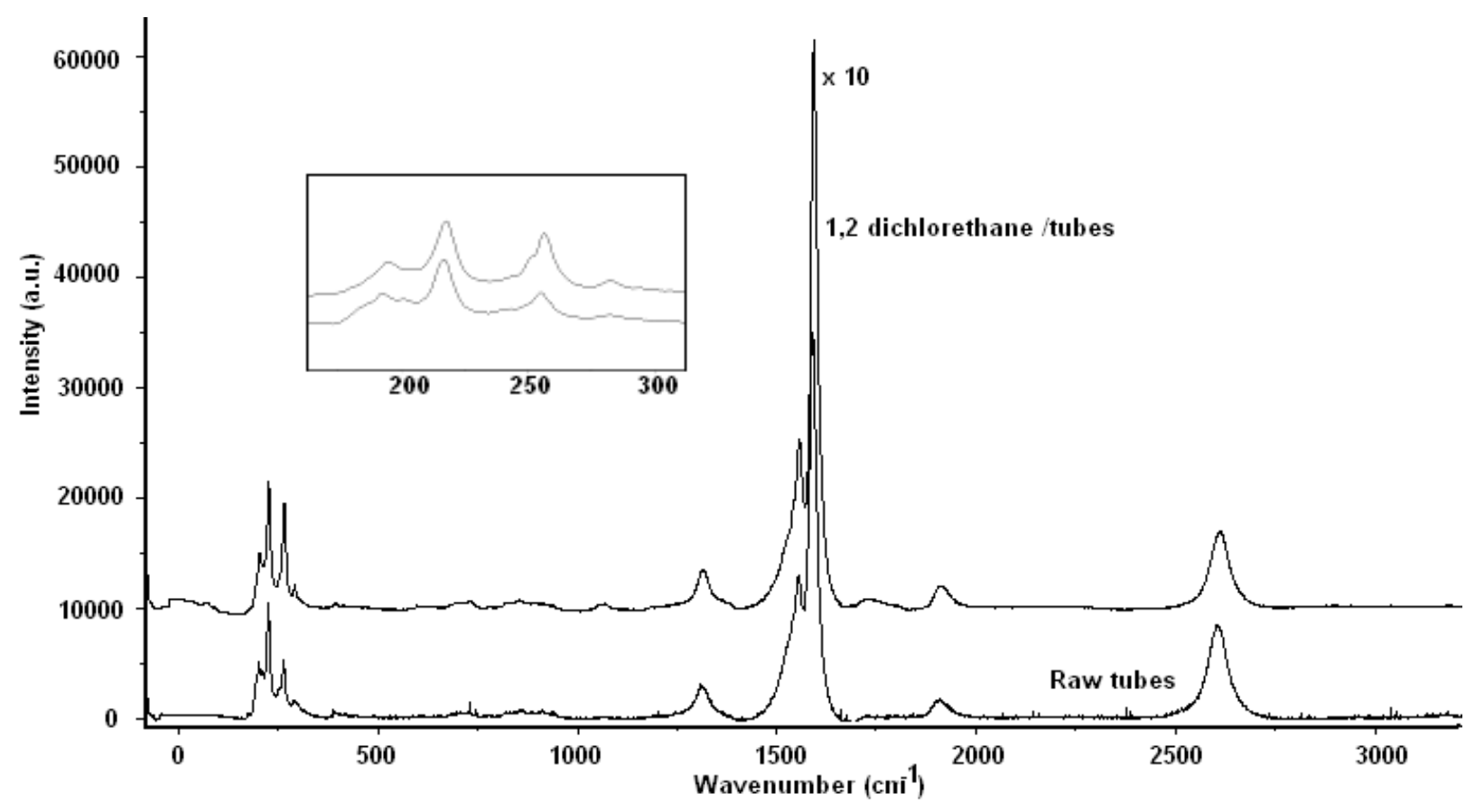

Fig 8: Stokes Raman spectra HiPco and DCE/ HiPco samples at 633nm inset of expanded $\mathrm{RBM}$ region

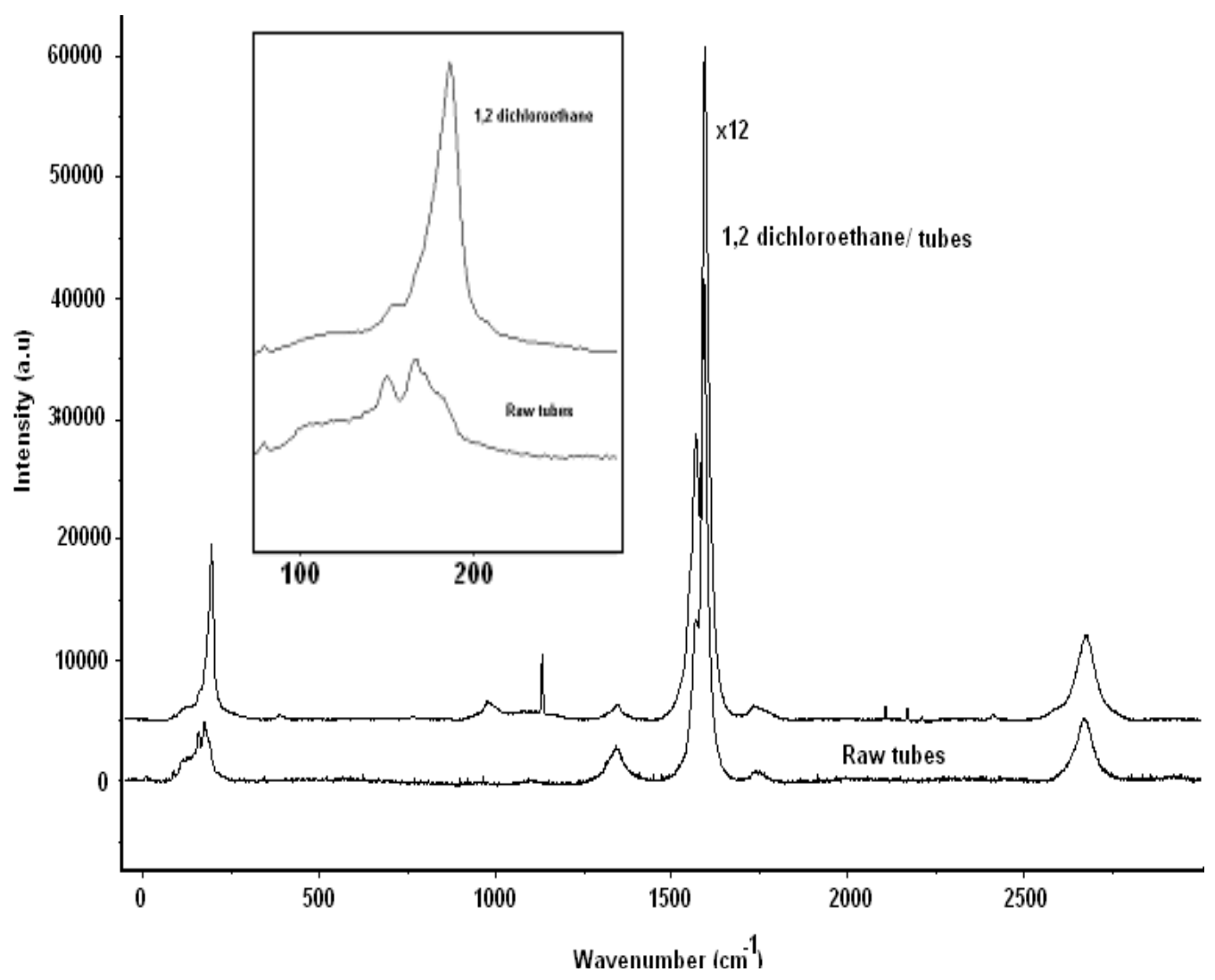

Fig. 9: Stokes Raman spectra arc-discharge and DCE / arc-discharge samples at $514 \mathrm{~nm}$ inset of expanded RBM region 


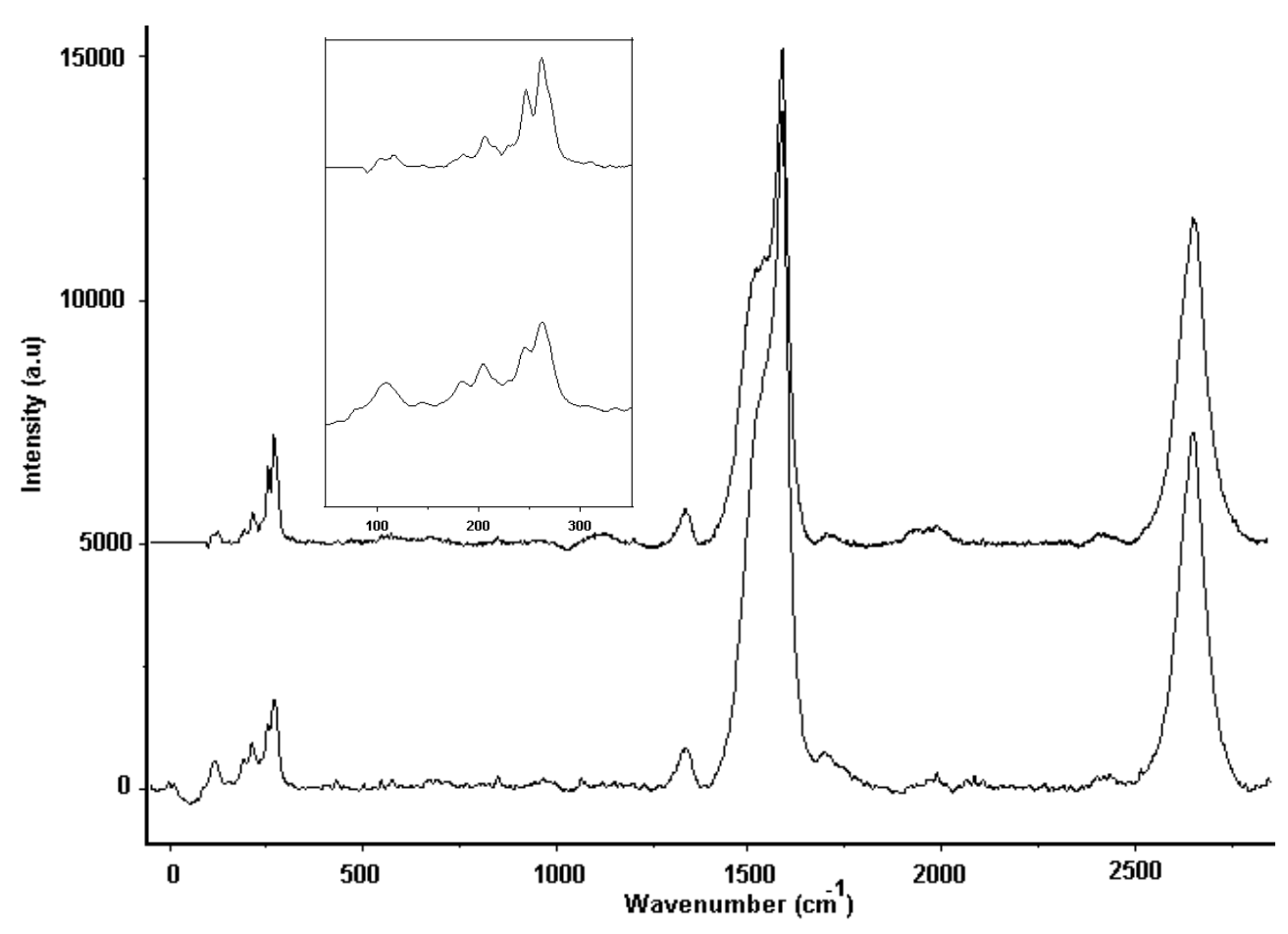

Fig. 10: Stokes Raman spectra of HiPco and DCE / HiPco samples at 514nm inset of the exnanded RRM reoinn

When the arc-discharge raw and treated samples are excited by the 514nm line (figure 9) there is no change to the profile of the G-line but again there is a significant change to the RBM's consistant with debundling.

For the HiPco at $514 \mathrm{~nm}$ there is some upshift in the RBM's but not as dramatic as for the arc-discharge samples. One interesting comment on the HiPco $514 \mathrm{~nm}$ samples; the G-line in the raw sample although predominantly semiconducting in character shows some metallic contribution in the $\omega_{G}^{-}$feature. In the DCE treated sample at $514 \mathrm{~nm}$ this metallic contribution seems enhanced further giving evidence to the DCE favouring metallic tubes.

The results from anaysis of the DMF treated samples show changes in the spectra intermediate between that of the DCE and the toulene.

\section{CONCLUSION}

There is evidence that DMF and DCE solubilise SWNT samples and produce some degree of debundling. Toulene shows no evidence of debundling however it does purifiy the arc-discharge tubes.The results from the DCE samples show the most promise. DCE seems to solubilize both tube types easily as evidenced from visual observation. However the degree of debundling is biggest for the arc-diacharge tubes. Furthermore the there is evidence that the DCE favours metallic tubes. Further work would look at decreasing the tube to solvent ratio to see if the level of debundling can be improved. Some form of microscopy is needed to measure the actual bundle size in each of the samples which can then be matched with the measured Raman shifts. Initial investigations using AFM have showed promise.

\section{REFERENCES}

[1] S.Iijima, Nature 354 , 56 (1991)

[2] R.Saito, G.Dresselhaus, M.S.Dresselhaus, Physical Properties of Carbon Nanotubes, Imperial College Press, London 1998 
[3] J.W.G.Wildöer, L.C.Venema, A.G.Rinzler, R.E.Smalley, and C.Dekker, Nature, 391,59(1998)

[4] T.W.Odom, J.L.Huang, P.Kim and C.M.Lieber, Nature, 385, 780(1997)

[5] T.W. Ebbesen and P. M. Ajayan, Nature,358, 220 (1992)

[6] S. Iijima and T. Ichihashi, Nature,363, 603 (1993)

[7] T.Guo, P.Nikolaev, A.Thess, R.E.Smalley Chem. Phys. Lett, 243, 49(1995)

[8] H.Dai, A.G. Rinzler, P.Nikolaev, A.Thess, D.T.Colbert and R.E.Smalley Chem. Phys. Lett, 260, 471(1996)

[9].W. Chiang, B.E. Brinson, A.Y. Huang, P.A. Willis, M.J. Bronikowski, R.E.Smalley J.L. Margrave and R.H. Hauge: J. Phys. B 105 , 8297-8301 (2001)

[10] P. Nikolaev , M.J. Bronikowski, R. Kelley Bradley, F Rohmund, D.T. Colbert, K.A. Smith, R.E. Smalley: Chem. Phys. Lett. 313 , 91-97 (1999)

[11] Strong, Anderson, Lafdi, Kuhn, Carbon 41,1477-1488 (2003)

[12] Thess et al , Science 273, 483-487 (1996)

[13] S.M. Keogh, T.G Hedderman, E.Gregan, G. Farrell, G. Chambers, H.J Byrne Journal of Phys. Chem. B 108 62336241(2004)

[14] E.Gregan, S.M. Keogh, T.G. Hedderman, G. Chambers, H.J. Byrne, Facility for Optical Characterisation and Spectroscopy (FOCAS), School of Physics, Dublin Institute of Technology, Kevin Street, Dublin 8, Ireland

[15]Chen, Saltiel, Manickavasagam, Schadler, Siegel, Yang, Journal of Colloid and Interface Science 208 91-97 (2004)

[16] A.Jorio, G. Dresselhaus, M.S.Dresselhaus, M.Souza, M.S.Dantas, M.A. Pimenta, A.M.Rao, R.Saito, C.Liu and H.M.Cheng, Phys.Rev.Lett.85, 2617-2620 (2000)

[17] S.D.M.Brown, A.Jorio, P.Corio, M.S.Dresselhaus, G. Dresselhaus, Phys.Rev.B, 63, 155414 (2001)

[18] H.Kataura, Y.Kumazawa, Y.Maniwa, I.Umezu, S.Suzuki, Y.Ohtsuka and Y.Achiba Synthetic Metals, 103, 2555(1999)

[19] S.D.M.Brown, A.Jorio, P.Corio, M.S.Dresselhaus, G. Dresselhaus, R.Saito, K.Kneipp, Phys.Rev.B-Rapid Comm (2001)

[20] M.A.Pimenta, J.H.Hafner, C.M.Lieber, R.Saito, Phys.Rev.B 65155412 (2002)

[21] M.S.Dresselhaus, P.C.Eklund, Adv.Phys.49 705 (2000)

[22] Jorio et al Phys.Rev.B 66115411 (2002)

[23] S.D.M.Brown, A.Jorio, P.Corio, M.S.Dresselhaus, G. Dresselhaus, Phys.Rev.B, 64, 073403 (2001)

[24] Pimenta, Hanlon, Marucci, Corio, Browne, Empedocles, Bawendi, G.Dresselhasu, M.Dresselhaus, Brazilian J.Phys.30 423 (2000)

[25] M.Dresselhaus, Jorio, Souza, G.Dresselhaus, Saito Physica B 323 15-20 (2002)

[26] A.M. Rao, E. Richter, S. Bandow, B. Chase, P.C. Eklund, K.A. Williams,S.Fang, K.R. Subbaswamy, M. Menon, A. Thess, R.E. Smalley, G. Dresselhaus and M.S.Dresselhaus, Science, 275, 187 (1997).

[27] Y.F.Wang, X.W Cao, S.F.Hu, Y.Y.Liu, G.X.Lan Chem.Phys.Lett.336 , 47-52 (2001)

[28] Rao, Chen, Richter, Schlecht, Eklund, Haddon, Venkateswaran, Kwon, Tománek, Phys.Review Letters 8617 (2001)

[29] Duesberg et al Phys. Review Letters 855436 (2000)

[30]Coleman, Fleming, Maier, O Flaherty, Minnett, Ferreira, Hutzler, Blau, J Phys.Chem.B.108,3446 (2004)

*Elizabeth.Gregan@dit.ie, phone +35314024736 